1. Lusis, A.J. 2000. Atherosclerosis. Nature. 407:233-241. 2. Castrillo, A., and Tontonoz, P. 2004. Nuclear receptors in macrophage biology: at the crossroads of lipid metabolism and inflammation. Annu. Rev. Cell Dev. Biol. 20:455-480.

3. Marx, N., Duez, H., Fruchart, J.C., and Staels, B 2004. Peroxisome proliferator-activated receptors and atherogenesis: regulators of gene expression in vascular cells. Circ. Res. 94:1168-1178.

4. Chawla, A., et al. 2001. A PPARgamma-LXR-ABCA1 pathway in macrophages is involved in cholesterol efflux and atherogenesis. Mol. Cell. 7:161-171.

5. Li, A.C., et al. 2000. Peroxisome proliferator-activated receptor $\gamma$ ligands inhibit development of atherosclerosis in LDL receptor-deficient mice. J. Clin. Invest. 106:523-531.

6. Collins, A.R., et al. 2001. Troglitazone inhibits formation of early atherosclerotic lesions in diabetic and nondiabetic low density lipoprotein receptor-deficient mice. Arterioscler. Thromb. Vasc. Biol. 21:365-371.

7. Lee, C.H., et al. 2003. Transcriptional repression of atherogenic inflammation: modulation by PPARdelta. Science. 302:453-457.
8. Tordjman, K., et al. 2001. PPAR $\alpha$ deficiency reduces insulin resistance and atherosclerosis in apoE-null mice. J. Clin. Invest. 107:1025-1034.

9. Duez, H., et al. 2002. Reduction of atherosclerosis by the peroxisome proliferator-activated receptor alpha agonist fenofibrate in mice. J. Biol. Chem. 277:48051-48057.

10. Rubins, H.B., et al. 1999. Gemfibrozil for the secondary prevention of coronary heart disease in men with low levels of high-density lipoprotein cholesterol. Veterans Affairs High-Density Lipoprotein Cholesterol Intervention Trial Study Group. N. Engl. J. Med. 341:410-418.

11. Oliver, W.R., Jr., et al. 2001. A selective peroxisome proliferator-activated receptor delta agonist promotes reverse cholesterol transport. Proc. Natl. Acad. Sci. U. S. A. 98:5306-5311.

12. Tontonoz, P., Nagy, L., Alvarez, J.G., Thomazy, V.A. and Evans, R.M. 1998. PPARgamma promotes monocyte/macrophage differentiation and uptake of oxidized LDL. Cell. 93:241-252.

13. Chinetti, G., et al. 2001. PPAR-alpha and PPARgamma activators induce cholesterol removal from human macrophage foam cells through stimula- tion of the ABCA1 pathway. Nat. Med. 7:53-58.

14. Ricote, M., Li, A.C., Willson, T.M., Kelly, C.J., and Glass, C.K. 1998. The peroxisome proliferator-activated receptor-gamma is a negative regulator of macrophage activation. Nature. 391:79-82.

15. Li, A.C., et al. 2004. Differential inhibition of macrophage foam-cell formation and atherosclerosis in mice by PPAR $\alpha, \beta / \delta$, and $\gamma . J$. Clin. Invest. 114:1564-1576. doi:10.1172/JCI200418730.

16. Joseph, S.B., et al. 2002. Synthetic LXR ligand inhibits the development of atherosclerosis in mice. Proc. Natl. Acad. Sci. U. S. A. 99:7604-7609.

17. Tangirala, R.K., et al. 2002. Identification of macrophage liver $\mathrm{X}$ receptors as inhibitors of atherosclerosis. Proc. Natl. Acad. Sci. U. S. A. 99:11896-11901.

18. Venkateswaran, A., et al. 2000. Human white/ murine ABC8 mRNA levels are highly induced in lipid-loaded macrophages. J. Biol. Chem. 275:14700-14707.

19. Wang, N., Lan, D., Chen, W., Matsuura, F., and Tall, A.R. 2004. ATP-binding cassette transporters G1 and G4 mediate cellular cholesterol efflux to highdensity lipoproteins. Proc. Natl. Acad. Sci. U. S. A. 101:9774-9779.

\title{
Fusion of bone marrow-derived stem cells with striated muscle may not be sufficient to activate muscle genes
}

\author{
Giulio Cossu
}

Stem Cell Research Institute, DIBIT, Milan, Italy.

\begin{abstract}
Several studies have demonstrated the existence of pluripotent bone marrow-derived stem cells capable of homing to injured cardiac and skeletal muscle; however, there has been little evidence demonstrating the induction of tissue-specific endogenous genes in donor stem cells following engraftment. A new study in this issue reports an intriguing finding that raises additional concerns relating to stem cell plasticity and stem cell therapy in an already heated and controversial field. The study demonstrates that wild-type bone marrow-derived side population stem cells are indeed readily incorporated into both skeletal and cardiac muscle when transplanted into mice that lack $\delta$-sarcoglycan - a model of cardiomyopathy and muscular dystrophy. However, these cells fail to express sarcoglycan and thus to repair the tissue, which suggests that this stem cell population has limited potential for cardiac and skeletal muscle regeneration (see the related article beginning on page 1577).
\end{abstract}

Bone marrow-derived side population (BM-SP) stem cells have the ability to repopulate the hematopoietic system (1) and to colonize, at low frequencies, many different tissues, including skeletal (2) and cardiac muscle (3). The $\delta$-sarcoglycan-null

Nonstandard abbreviations used: BM-SP, bone marrow-derived side population.

Conflict of interest: The author has declared that no conflict of interest exists.

Citation for this article: J. Clin. Invest. 114:1540-1543 (2004). doi:10.1172/JCI200423733. mouse is a model of cardiomyopathy and muscular dystrophy (corresponding to a human limb-girdle myopathy) and develops microinfarcts in heart and skeletal muscle (4). Muscle cell death in this model is related to the lack of $\delta$-sarcoglycan, which causes loss of the other sarcoglycans $(\alpha, \beta$, and $\gamma)$ and in turn disassembly of the dystroglycan complex (Figure 1), which is composed of several proteins that link the cytoskeleton to the basal lamina and reduce membrane stress during contraction. Absence or reduction of the dystroglycan complex results in increased fragility of the membrane and increased chance of damage to the muscle cell. Resident cells capable of repairing injured skeletal muscle (satellite cells) are well characterized (5), and despite the long-held belief that heart cells cannot regenerate, evidence for the existence of cells with a similar reparative function in the heart has only begun to accumulate during the last 2 years $(6,7)$. This observation suggests that injured $\delta$-sarcoglycan-deficient tissues should recruit both local and blood-born stem cells that may contribute to regeneration.

In this issue of the JCI, Lapidos et al. (8) transplanted BM-SP stem cells from wild-type male mice into female $\delta$-sarcoglycan-null mice, and their results, consistent with those from previous studies of stem cell-mediated skeletal and cardiac muscle regeneration, demonstrated the presence of these donor cells, determined by the presence of a $\mathrm{Y}$ chromosome inside cardiac and skeletal muscle at the expected frequency. A fraction of the nuclei of these Y chromosome-positive donor cells was unequivocally shown to be present inside the cytoplasm of several differentiated car- 


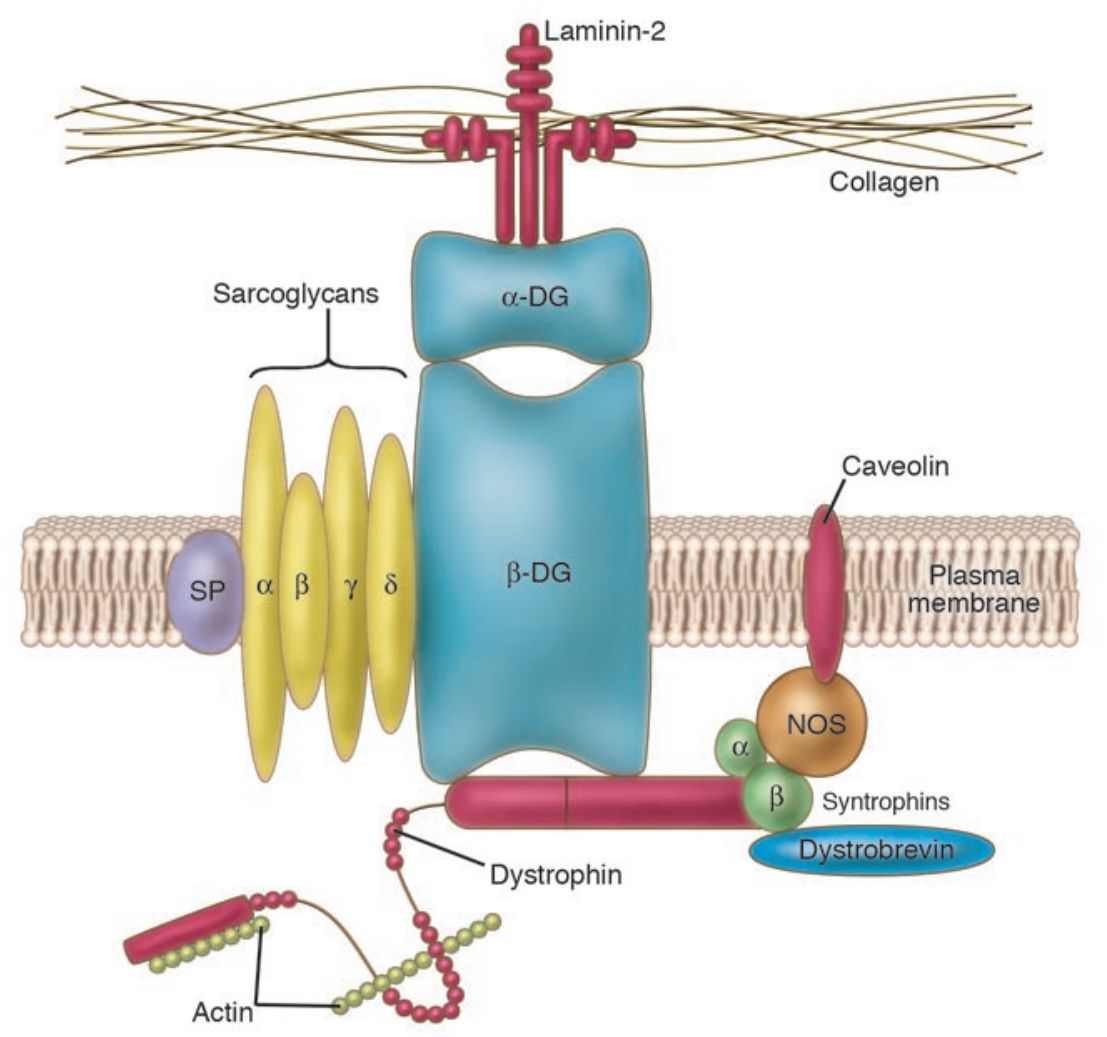

diomyocytes and myofibers. Surprisingly, exhaustive examination of the quadriceps muscles and of the hearts of the 14 recipient mice (representing many thousands of muscle cells) detected only 2 sarcoglycanpositive fibers; all cardiomyocytes containing donor-derived nuclei were sarcoglycan negative. The presence of only 2 sarcoglycan-positive fibers is important, as it confirms that BM-SP stem cells can produce $\delta$-sarcoglycan but do so at a negligible frequency. The absence of sarcoglycan expression from wild-type donor cells indicates impaired muscle differentiation of donorderived nuclei, thereby limiting the regenerative potential of these cells. Appropriate controls demonstrate that transplantation of primary myoblasts isolated and cultured from wild-type male mice into $\delta$-sarcoglycan-deficient female mice restore expression of all sarcoglycans in regenerated myofibers, which confirms that the sarcoglycan complex could be restored through transplantation in these mice. These data raise the question: Why do BM-SP stem cells fail to express $\delta$-sarcoglycan even after exposure to myogenic transcription factors?

\section{Why do these results apparently contrast with previous reports?}

The results of the Lapidos et al. (8) study tell us that the donor BM-SP stem cells used express the expected antigen repertoire, are able to reconstitute the hematopoietic system, and thus appear as "bona fide" BM-SP stem cells. The fact that they fail to produce the gene product ( $\delta$-sarcoglycan) absent in this myopathy and thus fail to ameliorate the phenotype is worrying and suggests that BM-SP stem cells may not be suitable for the cell therapy at least of cardiac and skeletal muscle. This stands in contrast to previous studies, which claimed tissue-specific differentiation of donor BM-SP stem cells occurred as these cells were detected inside skeletal muscle fibers (2) or cardiomyocytes (3). This conclusion was based on colocalization of donor cell-autonomous markers and tissue-specific gene products within the same muscle cell. The detection of $\delta$-sarcoglycan expression in a genetic model in which the $\delta$-sarcoglycan gene is missing clearly requires that the missing gene be derived exclusively from wild-type donor-derived nuclei. Lapidos and colleagues rightly point out that one or many host nuclei present within the same cytoplasm of cardiomyocytes or myofibers may have been the source of the tissue-specific gene products detected in previous studies and thus led to an erroneous conclusion. In other cases, dystrophin expression has been considered as evidence of muscle

\section{Figure 1}

Schematic representation of the dystroglycan complex at the membrane of the muscle cell. A number of transmembrane proteins, including dystroglycan (DG), sarcospan (SP), and sarcoglycans ( $\alpha, \beta, \gamma$, and $\delta)$, connect the actin cytoskeleton to the basal lamina by binding dystrophin (in turn linked to actin) on the cytoplasmic side and laminin on the extracellular side of the muscle plasma membrane. Other functionally important proteins such as nitric oxide synthetase (NOS) are maintained at the cytoplasmic side of the membrane by a different protein complex also bound to dystrophin.

gene expression by donor nuclei after transplantation in $m d x$ (i.e., dystrophindeficient) mice (a model of Duschenne muscular dystrophy) (2). However, it is known that in untransplanted $m d x$ mice, there is a population of revertant myofibers, which are capable of expressing dystrophin due to exon skipping (9). In other studies, researchers took advantage of the myosin light chain 1/3 fast (MLC1/3F) promoter, which drives LacZ expression only in striated muscle (10) and in normal developing muscle cells faithfully reflects expression of the endogenous myosin gene (11). The first study of skeletal muscle differentiation from unfractionated bone marrow-derived cells relied on the fact that this muscle-specific transgene can only be expressed by the donor-derived nuclei. It is still possible that in different experimental situations, transgenes may not reflect the correct level of expression of the endogenous genes; however, further studies will be necessary to determine the reason for the discrepancy between the results of studies showing the regenerative capability of this cell population and the observation, reported in this issue by Lapidos et al., that this capability is limited.

\section{Muscle differentiation is a multistep process}

We are inclined to think that once differentiation is triggered either by the binding of soluble molecules to the cell surface or by transcription factors from neighboring nuclei within the same cytoplasm (in the case of cell fusion), it will automatically lead to the full expression of the complete genetic repertoire of a differentiated tissue. Yet we know from decades of tissue culture studies that this is not true. In vitro, skeletal myoblasts differentiate into multinucleated myotubes upon depletion of growth factors in the medium and express a large number 

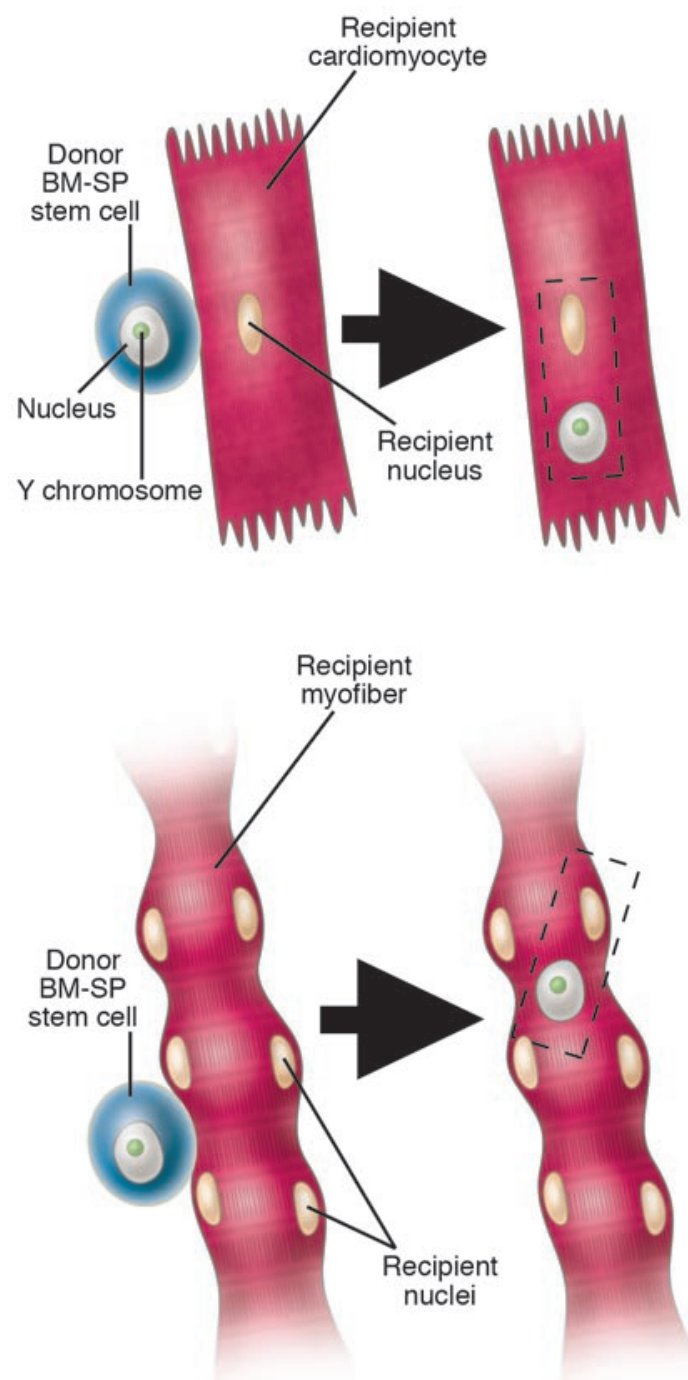

of muscle-specific genes (12). However the genes encoding adult isoforms of sarcomeric proteins as well as other "late" genes characteristic of mature muscle fibers in vivo are not expressed in vitro in the absence of nerve and hormones (13). $\delta$-Sarcoglycan is only expressed during the formation of the dystroglycan complex (Figure 1) and thus may represent a "late" muscle gene (14) that could not be expressed by BM-SP stem cell nuclei (Figure 2), possibly due to a failure to reprogram gene expression. From the experimental design of the Lapidos study (8), it was not possible to know to what degree the donor-derived nuclei have entered into the myogenic pathway (all muscle genes other than $\delta$-sarcoglycan may in fact derive from host nuclei) and whether $\delta$-sarcoglycan may be detectable at a later developmental stage, even though a longer time interval after transplantation was not sufficient to detect its expression.

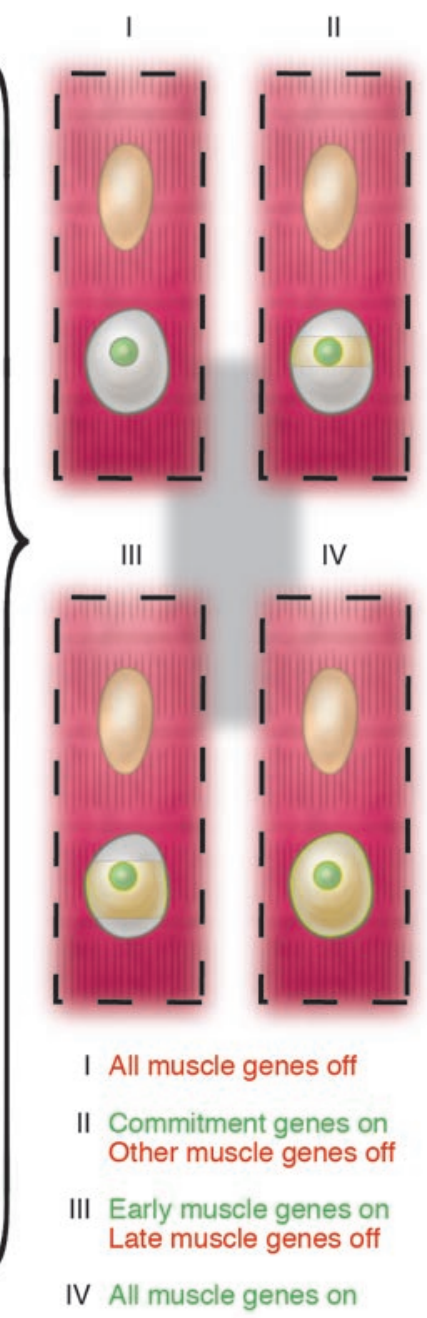

In this context it would be interesting to repeat the experiments conducted by Lapidos et al., obtaining BM-SP stem cells from mice that express a $\mathrm{LacZ}$ reporter gene under the control of a myogenic determination gene (i.e., $M y f 5$ or $M y o D$ ) or of a muscle gene expressed early after differentiation, such as $M L C 1 / 3 F(11)$. I would predict that LacZ would be expressed at variance with $\delta$-sarcoglycan. If this proves to be the case, then previous conflicting reports could be reconciled.

\section{Can BM-SP stem cells be used for therapy?}

Despite a number of problems such as low abundance, failure to grow extensively in vitro, and low differentiation potency toward nonhematopoietic lineages, BM-SP stem cells have become very popular in experimental stem cell therapy for primary myopathies and heart infarct (reviewed in

\section{Figure 2}

Different degrees of reprogramming of the nucleus of a wild-type BM-SP stem cell from a male mouse once inside a cardiomyocyte or a myofiber of a female $\delta$-sarcoglycan-null mouse. The model proposes that the $Y$ chromosome-positive (green) donor nucleus (gray) will be exposed to transcription factors from recipient nuclei (cream) as a consequence of either fusion or transdifferentiation. The donor nucleus may then be subject to different fates: no reprogramming at all (I); transcription of myogenic determination genes but not of muscle structural genes (II); transcription of myogenic determination genes and of muscle structural genes expressed during early differentiation only (III); or transcription of all muscle genes (IV). The last event is ruled out in the study of Lapidos et al. (8) by the lack of $\delta$-sarcoglycan expression observed after transplantation of wild-type BM-SP stem cells into $\delta$-sarcoglycan-null mice. refs. 15 and 16). However, if their differentiation toward a mature muscle phenotype cannot be achieved, their prospective use in stem cell therapy for the regeneration of both cardiac and skeletal muscle becomes unrealistic. On the other hand, it should be considered that chromatin remodeling agents, which epigenetically modify histones and associated proteins, may contribute to enhancing expression of certain genes such as those associated with muscle maturation. This hypothesis would be easily testable by repeating the experiments in mice treated with histone deacetylase or methyl-transferase inhibitors.

Finally, it would be important to repeat the same experiments described here by Lapidos et al. (8) with other types of stem cells, such as mesenchymal stem cells (17), multipotent adult progenitors (18), or even neural stem cells (19). None of these cell types have been tested in models of muscu- 
lar dystrophy, where replacement of specific gene products such as the sarcoglycans could easily be detected. Mesoangioblasts, on the other hand, have been shown to be efficacious in restoring expression of $\alpha$-sarcoglycan in the $\alpha$-sarcoglycan-null mouse as well as the expression of the whole dystrophin complex, including $\delta$-sarcoglycan (20). All of these cell types are easy to expand in culture and, once their homing and muscle differentiation activities can be optimized, they may represent a better perspective for the stem cell therapy of striated muscle diseases than BM-SP stem cells.

\section{Acknowledgments}

I would like to thank G.L. Condorelli and M. Sampaolesi for critical reading of the manuscript.

Address correspondence to: Giulio Cossu, Stem Cell Research Institute, DIBIT, H. San Raffaele, 58 Via Olgettina, 20132 Milan, Italy. Phone: 3902-2156-0250; Fax: 39022156-0220; E-mail: cossu.giulio@hsr.it.
1. Goodell, M.A., et al. 1996. Isolation and functional properties of murine hematopoietic stem cells that are replicating in vivo. J. Exp. Med. 183:1797-1806.

2. Gussoni, E., et al. 1999. Dystrophin expression in the mdx mouse restored by stem cell transplantation. Nature. 401:390-394

3. Orlic, D., et al. 2001. Bone marrow cells regenerate infarcted myocardium. Nature. 410:701-705.

4. Coral-Vazquez, R., et al. 1999. Disruption of the sarcoglycan-sarcospan complex in vascular smooth muscle: a novel mechanism for cardiomyopathy and muscular dystrophy. Cell. 98:465-474.

5. Seale, P., and Rudnicki, M.A. 2000. A new look at the origin, function, and "stemcell" status of muscle satellite cells. Dev. Biol. 218:115-124.

6. Beltrami, A.P., et al. 2003. Adult cardiac stem cells are multipotent and support myocardial regeneration. Cell. 114:763-776.

7. Oh, H., et al. 2003. Cardiac progenitor cells from adult myocardium: homing, differentiation, and fusion after infarction. Proc. Natl. Acad. Sci. U. S. A. 100:12313-12318.

8. Lapidos, K.A., et al. 2004. Transplanted hematopoietic stem cells demonstrate impaired sarcoglycan expression after engraftment into cardiac and skeletal muscle J. Clin. Invest. 114:1577-1585. doi:10.1172/JCI200423071.

9. Lu, Q.L., et al. 2000. Massive idiosyncratic exon skipping corrects the nonsense mutation in dystrophic mouse muscle and produces functional revertant fibers by clonal expansion. J. Cell Biol. 148:985-996.

10. Ferrari, G., et al. 1998. Skeletal muscle regeneration by bone marrow derived myogenic progenitors. Science. 279:1528-1530.

11. Kelly, R., et al. 1995. Myosin light chain 3F regulatory sequences confer regionalised cardiac and skeletal muscle expression in transgenic mice. J. Cell Biol. 129:383-396.

12. Arnold, H.H., and Winter, B. 1998 Muscle differentiation: more complexity to the network of myogenic regulators. Curr. Opin. Genet. Dev. 8:539-544.

13. Weiss, A., and Leinwand, L.A.1996. The mammalian myosin heavy chain gene family. Annu. Rev. Cell Dev. Biol. 12:417-439.

14. Liu, L.A., and Engvall, E. 1999. Sarcoglycan isoforms in skeletal muscle. J. Biol. Chem. 274:38171-38176.

15. Cossu, G., and Sampaolesi, M. 2004. New therapies for muscular dystrophy: cautious optimism. Trends Mol. Med. 10:516-520

16. Itescu, S., Schuster, M.D., and Kocher, A.A. 2003. New directions in strategies using cell therapy for heart disease. J. Mol. Med. 81:288-296.

17. Liechty, K.W., et al. 2000. Human mesenchymal stem cells engraft and demonstrate site specific differentiation after in utero transplantation in sheep. Nat. Med. 6:1282-1286.

18. Reyes, M., et al. 2001. Purification and ex vivo expansion of postnatal human marrow mesodermal progenitor cells. Blood. 98:2615-2625.

19. Galli, R., et al. 2000. Skeletal myogenic potential of adult neural stem cells. Nat. Neurosci. 3:986-991.

20. Sampaolesi, M., et al. 2003. Cell therapy of alpha sarcoglycan null dystrophic mice through intraarterial delivery of mesoangioblasts. Science. 301:487-492.

\title{
Marfan syndrome and mitral valve prolapse
}

\author{
Arthur E. Weyman and Marielle Scherrer-Crosbie
}

Cardiac Ultrasound Laboratory, Cardiology Division, Department of Medicine, Massachusetts General Hospital, Boston, Massachusetts, USA.

\begin{abstract}
Mitral valve prolapse (MVP), an abnormal displacement into the left atrium of a thickened and redundant mitral valve during systole, is a relatively frequent abnormality in humans and may be associated with serious complications. A recent study implicates fibrillin-1, a component of extracellular matrix microfibrils, in the pathogenesis of a murine model of MVP (see the related article beginning on page 1586). This investigation represents an initial step toward understanding the mechanisms involved in human MVP disease and the development of potential treatments.
\end{abstract}

\section{Mitral valve prolapse: scope of the problem and evolution of the defining criteria}

Mitral valve prolapse (MVP) is generally understood to be the displacement of abnormally thickened, redundant mitral leaflet(s) into the left atrium during systole (Figure 1) (1). One of the possible consequences of this condition is that the malfunctioning mitral valve allows back-

Nonstandard abbreviations used: FBN1, fibrillin-1; MVP, mitral valve prolapse; TGFBR2, TGF- $\beta$ receptor 2 .

Conflict of interest: The authors have declared that no conflict of interest exists.

Citation for this article: J. Clin. Invest. 114:1543-1546 (2004). doi:10.1172/JCI200423701. flow of blood in the left atrium (mitral regurgitation), which, when severe, leads to left ventricular enlargement and failure. Besides severe mitral regurgitation, MVP has been associated with serious complications such as bacterial endocarditis and sudden death, and primary mitral valve surgery is currently performed most frequently to specifically treat $\operatorname{MVP}(1,2)$. Since the early 1970s, research has suggested that echocardiography is the ideal noninvasive technique to visualize the prolapsing mitral leaflets (3-5). However, continually changing echocardiographic techniques and criteria for the diagnosis of MVP have in many cases further obscured, rather than clarified, our understanding of prolapse in its primary form and in association with other disorders (6). Over the past decade, new echocardiographic criteria for MVP have been established based on an understanding of the 3D structure of the mitral valve (7). Defined according to these criteria, prolapse is the displacement of 1 or both mitral leaflets by more than $2 \mathrm{~mm}$ above the high points of the mitral annulus as recorded in either the parasternal or apical long-axis view (Figure 1 , detail). This $2 \mathrm{~mm}$ displacement derives from studies showing that smaller displacements are not associated with increased leaflet thickness, mitral regurgitation, or valve-related complications (8). In cases where leaflet displacement is greater than $2 \mathrm{~mm}$, prolapse is further subdivided into classic and nonclassic forms based on leaflet thickness (classic, $>5 \mathrm{~mm}$; and nonclassic, $\leq 5 \mathrm{~mm}$ ), with complications such as endocarditis or severe mitral regurgitation generally occurring in patients with classic prolapse (9).

Using these criteria, a recent population study of 3,491 subjects from the offspring 